\title{
Análisis de los flujos audiovisuales: El cine en México
}

\author{
Francisco Javier Martínez Garza \\ ITESM Campus Monterrey \\ CINCO
}

\section{Introducción:}

En su artículo 4, la actual Ley de Cinematografía [1] resalta que esta industria es un vehículo de expresión artística y educativa, y hace hincapié en que el cine, "constituye una actividad cultural primordial". Más adelante en el artículo 14 de la misma, se vuelve a reafirmar que la producción cinematográfica nacional constituye una actividad de interés social... por expresar la cultura nacional y contribuir a fortalecer los vínculos de identidad nacional entre los diferentes grupos que la conforman. No obstante, en la realidad son cada vez menos la cantidad de películas nacionales que se producen y se distribuyen en México.

Al respecto, Víctor Ugalde, Secretario Técnico de Fidecine, ha señalado que a diferencia de lo que sucedía anteriormente, cuando la producción promedio anual en México superaba las cien películas, durante los últimos años las carteleras cinematográficas se encuentran prácticamente inundadas de cintas que provienen de otras naciones, principalmente de los Estados Unidos de Norteamérica[2]. Sobre este mismo punto, Alfredo Joskowicz señaló que el año de 2002, fue desastroso para la industria fílmica nacional, debido a que sólo se produjeron 14 cintas contra los 21 del año 2001 o las 28 del 2000 [3]. Sobre esa situación, el investigador Enrique Sánchez Ruíz sostiene que la industria del cine mexicano se ha ido sumiendo en su peor crisis, en medio de algunos pequeños triunfos de crítica y otros pocos de taquilla [4]. Este mismo autor ha señalado que tanto la producción, como la distribución y la exhibición de la industria cinematográfica mexicana está pasando por un intenso proceso de contracción, concentración y transnacionalización [5].

La participación que ha tenido el Gobierno del país en torno al cine, se ha dado de muy distintas formas, más lo cierto es que las reglas o políticas implementadas no han fortalecido lo suficiente a esta industria cultural. Las políticas implementadas en este ramo por 
cada una de las administraciones gubernamentales han sido incluso diametralmente opuestas. Así mientras que durante el gobierno del presidente Luis Echeverría, la tendencia fue a la estatización -aunque no necesariamente nacionalizada- [6], con Carlos Salinas de Gortari, la tendencia fue totalmente inclinada hacia la liberación del mercado a través de la Ley de Cinematografía de 1992 [7]: Esta ley causó una profunda indignación entre los cineastas, académicos, intelectuales e incluso políticos mexicanos, dado que las mismas, a juicio de ellos, llevaría al cine mexicano a su total destrucción. Finalmente con base en las presiones ejercidas por éstos, la Ley de Cinematografía de 1992, fue reformada por el pleno del Congreso.

La modificación a dicha ley resultó más difícil de lo esperado, sobre todo por la fuerte oposición de quienes hasta entonces se habían beneficiado de la misma. "En contra de este grupo se movilizaron los grandes intereses económicos de las empresas norteamericanas, mismas que en el ' 98 controlaron más del $85.0 \%$ de las pantallas nacionales, dejándole un ridículo $2 \%$ a las cintas mexicanas y el 13 $\%$ restante a la cinematografía mundial" [8].

La situación anterior es tan sólo uno de los resultados que se pueden generar como resultado de la poca atención que las autoridades mexicanas han venido prestando a la industria cinematográfica. En ese sentido las grandes empresas transnacionales de cine son quienes en verdad se vienen beneficiado del mercado nacional desde hace ya muchos años. La gran penetración que ha conseguido el cine norteamericano a nivel mundial se puede ubicar, según Murciano, casi desde el inicio de la segunda década del siglo pasado. Murciano señala que para mediados de la década de los 30’s, "el cine se había convertido en una de las más importantes y florecientes industrias americanas, y el control de los grandes estudios se hallaba concentrado directa o indirectamente en manos de los grupos financieros más poderosos de los Estados Unidos [9].

Teniendo como base de lanzamiento la Motion Pictures Association of America (MPAA), los estudios norteamericanos han instaurado una gran red de distribución a nivel mundial defendiendo en todo momento los intereses de sus agremiados y oponiéndose a quienes van en contra de sus intereses expansionistas. De tal manera que cuando los legisladores mexicanos autorizaron en el año $2002 \mathrm{el}$ incremento de 1 peso a la taquilla, a fin de destinar el mismo al apoyo de la producción cinematográfica en México. Jack Valenti, presidente de la MPAA, se manifestó en contra de la medida e incluso mandó 
una carta al presidente Vicente Fox [10]. En el fondo, el malestar de Valenti, tenía que ver con su temor a las medidas legislativas, las cuales a su juicio podrían afectar los intereses del cine norteamericano en México.

La defensa que hacen de lo que consideran "su mercado" las organizaciones estadounidenses tiene que ver con la importancia que representa la taquilla en México, dado que el año 2002 estuvo respaldada por la asistencia de cerca de 150 millones de personas [11]. El promedio de asistencia a las salas indican que en México por pantalla acuden al cine 85,000 personas. Esta cifra es muy considerable, sobre todo si se considera que en los Estados Unidos de Norteamérica dicho promedio se encuentra en los 33,000. De ahí que los inversionistas transnacionales sigan expandiéndose en el país. Tan sólo para este año del 2003, se espera que entre 120 y 150 salas más, se integren al mercado actual [12].

Por otra parte, resulta por demás increíble que la distribución del cine en nuestro país esté controlada prácticamente por los grandes consorcios norteamericanos representados por Columbia, United International Pictures, Fox y Warner [13]. El desequilibrio en la oferta de cine ya había sido analizada desde principio de los años 80 's por Tapio Varis y Gubak, quienes concluyeron que la exhibicion de las películas en buena parte de las pantallas del mundo, dependía de unas cuantas compañías trasnacionales, las cuales operan desde los Estados Unidos [14]. La fortaleza de estas grandes empresas del cine es por mucho superior a la mejor de este país, no obstante, lo menos que podemos esperar de nuestras autoridades es que al menos tomen conciencia de la importancia cultural y económica que representa la industria cinematográfica. Por lo pronto, esperamos que al menos la actual Ley de Cinematografía sea acatada por los distribuidores de cine que operan en el país, todo ello con la finalidad de sacar al cine mexicano del marasmo en el que se encuentra actualmente.

\section{Planteamiento del problema:}

Considerando los puntos que se han señalado en la introducción del trabajo, es que el presente estudio se propone conocer e identificar la oferta y la procedencia cinematográfica que se exhibe en las ciudades de México y Monterrey, Nuevo León. A fin de conocer la situación que guarda la oferta de cine nacional. 
En ese sentido se han planteado las siguientes preguntas de investigación:

(a) ¿En qué medida participan las películas originales del cine mexicano en la cartelera cinematográfica?

(b) Excluyendo a las películas mexicanas, ¿de qué país o de que países proceden las cintas que se difunden en la cartelera cinematográfica?

(c) ¿Existe alguna diferencia entre el tiempo de permanencia en cartelera de las películas mexicanas y de aquellas que provienen de otras naciones?

(d) ¿En qué región del mundo se están originando las películas que se presentan en las ciudades incluidas en la muestra?

(e) ¿Cuáles son los estudios cinematográficos que más películas proveen a las salas de exhibición en las ciudades incluidas en la muestra?

\section{Método de trabajo:}

En la realización del estudio se recurrió a la técnica del análisis de contenidos, seleccionándose como unidad de análisis a la cartelera cinematográfica de las ciudades de Monterrey y México. Se consideró analizar la oferta que se presenta en estas ciudades, debido a la cantidad de salas con que cuenta cada una de éstas, las cuales les convierte junto a las ciudades de Veracruz y de Guadalajara, en las que cuentan con la mayor cantidad en el país [15]. Con base en ello, es de presuponerse que la mayoría de las cintas que son ofertadas en el país, tienen una alta probabilidad que éstas sean o hayan sido presentadas en las pantallas de ambas ciudades.

La información se obtuvo de diarios matutinos instalados en cada una de las ciudades analizadas. En el caso de Monterrey, ésta se obtuvo de la cartelera cinematográfica que apareció publicada en el matutino El Norte. Mientras que en el análisis realizado a las carteleras de la ciudad de México se recurrió al matutino Reforma.

El estudio abarca una extensión de los cuatro primeros meses del año 2003, iniciándose la recolección cada día lunes a partir del 6 de enero hasta concluir el 28 de abril. En caso de que no se contara con el matutino del día lunes, se buscaba la información en el diario del siguiente día. El proceso que se siguió en el levantamiento de la información, concluyó finalmente con una muestra que comprende 17 semanas naturales de la cartelera cinematográfica de cada una de las ciudades contempladas. La decisión de realizar el levantamiento 
de información una vez por semana tiene que ver con la propuesta legislativa en materia cinematográfica de México, según la cual en su artículo 19 la oferta de la cartelera deberá mantenerse como mínimo durante el mencionado espacio temporal [16].

Con la finalidad de estandarizar la codificación, a la vez que evitar errores en la misma, se recurrió a la página electrónica de All Movie [17], en donde se brinda una detallada descripción de la mayoría de las cintas que son exhibidas en el país, independientemente del origen que tengan las mismas. Por consiguiente, las personas que participaron en la codificación de la información, se vieron en la necesidad de recurrir a dicha página electrónica. En aquellos casos, cuando no se pudo localizar ninguna referencia a una película, se recurrió a la información técnica que se localizaba en los periódicos seleccionados.

\section{Análisis de los Resultados:}

Durante los cuatro primeros meses del año en las ciudades seleccionadas se presentaron un total de 161 películas diferentes. Esta cantidad, incluye la suma de cintas que se presentaron en ambas ciudades, puesto que muchas de ellas que aparecieron publicadas en la cartelera de cine de la ciudad de México, no fueron incluidas en la cartelera regiomontana o viceversa. De los 161 títulos distintos de películas, en la ciudad de Monterrey se presentaron 116, mientras que en la ciudad de México aparecieron 148 títulos diferentes.

La mayoría de las producciones que fueron exhibidas son de reciente creación. De tal manera que 6 de cada 10 cintas habían sido realizadas durante el año 2002 . El $1 \%$, fueron realizadas justo durante el año 2003 y casi el $2 \%$ correspondió a cintas producidas durante el año 2001. Esto significa que sólo 1 de cada diez películas exhibidas contaba con más de 3 años de antigüedad.

Entre las películas exhibidas se destaca la presencia de una buena variedad de géneros cinematográficos. Sin embargo, entre todos ellos destaca debido a la cantidad de cintas que sobre el mismo se exhibieron, las películas relacionadas con el género dramático. Este género, al igual que los géneros de comedia y de aventuras (Tabla 1) son los que más participación tuvieron durante el periodo del estudio. 


\begin{tabular}{|ll|}
\hline Tabla 1 & \\
Género de la película & $\%$ \\
Drama & 29 \\
Comedia & 17 \\
Aventuras & 14 \\
Acción & 10 \\
Film Histórico & 6 \\
Romance & 6 \\
Misterio & 6 \\
Terror /Horror & 5 \\
Tragicomedia & 4 \\
Ciencia Ficción & 2 \\
Musical & 1 \\
Total & 100 \\
& $(\mathrm{n}=161)$ \\
\hline
\end{tabular}

Tabla 1 Aún y cuando se pudiera hablar de una amplia variedad de géneros en las carteleras cinematográficas, lo cierto es que casi una de cada tres cintas pertenecen al género drama.

En términos proporcionales, una de cada tres cintas tenían que ver precisamente con el género de "drama". Entre las películas que se relacionan con este género destaca "Frida". Esta cinta norteamericana en donde se aborda la vida de la pintora mexicana, fue una de las que mayor tiempo permaneció en pantalla. considerando las semanas que se exhibió en la ciudad de Monterrey, así como las semanas que permaneció en las carteleras de la ciudad de México, "Frida" sumó 24 semanas en pantalla. Otros filmes que también forman parte de este mismo género y que se mantuvieron por una buena cantidad de tiempo en la cartelera fueron: "Hable Con ella" (20 semanas) y, "Las Horas" (18 semanas).

Tabla 2

\begin{tabular}{|l|l|l|}
\hline 1 & Películas & Semanas en pantalla \\
1 & Harry Potter y la cámara secreta & 24 \\
2 & Frida & 24 \\
& El señor de los anillos. Las dos torres & 23 \\
\hline
\end{tabular}




\begin{tabular}{|l|l|l|}
\hline 2 & El planeta del tesoro & 23 \\
3 & Los mini campeones & 20 \\
3 & Hable con ella & 20 \\
3 & Casarse está en griego & 20 \\
4 & El aro & 18 \\
4 & Las Horas & 18 \\
5 & Chicago & 17 \\
5 & El hijo de la novia & 17 \\
6 & Atrápame si puedes & 16 \\
6 & Recién casados & 16 \\
7 & Lucía y el sexo & 15 \\
7 & 8 Mile, calle de las ilusiones & 15 \\
8 & Pandillas de Nueva York & 14 \\
8 & Confesiones del señor Schmidt & 14 \\
8 & El Rey León & 14 \\
8 & Ladrón de orquideas & 14 \\
8 & El experimento & 14 \\
8 & Lee mis labios & 14 \\
8 & Amor a segunda vista & 14 \\
9 & La hija del canibal & 13 \\
10 & Canguro Jack & 12 \\
10 & Amar te duele & 12 \\
10 & Analízate & 12 \\
\hline
\end{tabular}

Tabla 2 Relación de películas que se mantuvieron durante un mayor período de tiempo en la cartelera cinematográfica.

Aún y cuando la cantidad de películas de comedia que se exhibieron no es tan grande como las de género dramático, éstas tuvieron también una nutrida participación en las carteleras cinematográficas durante los primeros cuatro meses del 2003. Entre las películas que pertenecen a este último género, sobresalen, "Los mini campeones" y "Casarse está en Griego" (20 semanas c/u), así como también "El hijo de la novia" (17 semanas).

En la cartelera cinematográfica también aparecieron con cierta regularidad las películas relacionadas con el género de aventuras. Entre las cintas que pertenecen a este género y que se mantuvieron durante un buen período de tiempo en cartelera se encuentra "Harry Potter y 
la cámara secreta", la cual se mantuvo por espacio de 24 semanas. También, "El señor de los anillos: Las dos torres", la cual se presentó en los cines de las ciudades estudiadas durante 23 semanas consecutivas.

De acuerdo con el estudio, siete de cada diez películas exhibidas durante el proceso de investigación, tienen que ver con los géneros de drama, de aventuras, de comedia o de acción. Así mismo, es importante destacar que las cintas relacionadas con estos géneros, no sólo son las que cubren la mayor cantidad en la cartelera, sino que también son las que permanecen durante una mayor cantidad de tiempo en ella. En las carteleras cinematográficas, también es posible localizar otro tipo de géneros distintos a los que se han mencionado. Sin embargo, la cantidad de cintas relacionadas con los mismos se encuentra muy por debajo de géneros mencionados.

La mayor parte de las películas que se exhibieron en los cines de Monterrey y en la ciudad de México, fueron clasificadas como propias para adolescentes (B), de tal forma que la mayoría de las cintas pueden ser vistas con excepción de los niños, por todo tipo de personas. De hecho, en la programación analizada, se aprecia que existen muy pocas películas dedicadas exclusivamente al mercado de los adultos. De acuerdo con los resultados del estudio, sólo el $1 \%$ de la totalidad de cintas que se exhibieron, estaban enfocadas exclusivamente en dicho segmento. Lo anterior, invita a reflexionar en torno al interés que tienen las empresas cinematográficas por cubrir la mayor cantidad de público con cada una de sus producciones.

Además de presentar el más nutrido número de películas, también las cintas clasificadas como propias del público infantil y adolescente, fueron las que permanecieron durante una mayor cantidad de tiempo en cartelera. Al respecto, 7 de las cintas clasificadas propias del público infantil, esto es, aquellas que pueden ser vistas por todo tipo de público, se mantuvieron en cartelera durante más de 20 semanas, en comparación a sólo 1 cinta, que fue clasificada como propia del mercado adulto, y la cual se mantuvo por un espacio de 18 semanas en cartelera.

De acuerdo a lo anterior, 2 de cada 3 cintas que fueron exhibidas en las ciudades analizadas, buscan cubrir prácticamente la totalidad del público. Así mismo, son justo las producciones destinadas al público adolescente e infantil -en ese orden-, las que más tiempo permanecieron en la cartelera cinematográfica, tal y como ya se ha mencionado antes (tabla 2 ). 


\subsection{Procedencia de las cintas:}

Las carteleras cinematográficas refleja la participación de un grupo muy reducido de naciones en lo que se ha denominado la industria cinematográfica. Pues si bien es cierto que las películas que se exhibieron provenían de 27 distintos países del mundo, sólo 10 de ellos participan por sí solos. El resto participan más bien en coproducciones, en donde toman parte 2 o más países. De igual forma, del total de países cuyas cintas se exhibieron en México durante este período, en realidad sólo fueron 7 los que sobresalen. Cuatro de los cuales pertenecen a la Unión Europea. En ese sentido, la participación que tienen las naciones europeas es considerable, sobre todo si se considera que una de las naciones, en este caso Francia, participó con una mayor cantidad de películas incluso que México.

No obstante, fueron los Estados Unidos de Norteamérica quienes participaron con la mayor cantidad de películas. Por sí mismo, esta última nación participó del $47 \%$ del total de cintas que fueron exhibidas. La diferencia porcentual que existe entre el vecino país del norte, y la nación que le sigue en cuanto la cantidad de oferta en cartelera, en este caso con Francia, es de 35 puntos porcentuales (tabla 3). Lo cierto es que la mayoría de ellas fueron realizadas en los Estados Unidos de Norteamérica. Los Estados Unidos de Norteamérica, son la nación que tiene actualmente la mayor cantidad de películas en exhibición en este país. Su participación es tal, que incluso por si misma esta nación dobla en cantidad, al total de las películas que provienen de la Unión Europea.

México, aparece también entre las naciones que participaron con una mayor cantidad de películas. Sin embargo, la diferencia porcentual que existe en la cantidad de películas que se produjeron en México y las que se produjeron en los Estados Unidos de Norteamérica es bastante grande. En las salas cinematográficas de Monterrey y de la ciudad de México fueron exhibidas 17 películas originales de este país. De éstas, 12 fueron realizadas exclusivamente por cineastas mexicanos y 5 se realizaron en colaboración con otras naciones. Principalmente con España. 


\begin{tabular}{|l|l|l|l|}
\hline Tabla 3 & & & \\
\hline País & Producciones & Coproducciones & Total \\
USA & 67 & 10 & 77 \\
Francia & 7 & 13 & 20 \\
México & 12 & 5 & 17 \\
Alemania & 4 & 10 & 14 \\
España & 5 & 8 & 13 \\
Reino Unido & 3 & 8 & 11 \\
Canadá & 1 & 4 & 5 \\
\hline
\end{tabular}

Tabla 3 En la cartelera cinematográfica se aprecia la participación de una buena cantidad de paises de la Unión Europea como Francia, Alemania y España. Sin embargo, la mayoría de las cintas que se exhibieron procedían de los estados Unidos de Norteamérica.

Alemania, España y el Reino Unido, son países miembros de la Unión Europea que también exhibieron sus obras durante el período de análisis. Incluso, la participación que tiene cualquiera de estas 3 naciones supera a la que tiene Canadá. Sin embargo, si se ubica a las naciones que participaron en la cartelera cinematográfica de las ciudades analizadas, en base al bloque comercial al cual pertenece, es posible reparar que 9 de cada 10 cintas, provienen sólo de los bloques integrados por las naciones de América del Norte (NAFTA) y a las de la Unión Europea (UE), destacándose la ausencia o casi nula participación de películas producidas en otros sitios del orbe.

También se destaca en el análisis -como ya se ha comentado-, la poca presencia del cine mexicano en las carteleras cinematográficas. Pues aún y cuando la región de América del Norte tiene la supremacía en cuanto ser el origen de la mayoría de las películas que se presentan, lo cierto es que la mayoría de ellas fueron realizadas en los Estados Unidos de Norteamérica. Los Estados Unidos de Norteamérica, son la nación que tiene actualmente la mayor cantidad de películas en exhibición en este país. Su participación es tal, que incluso por si misma esta nación dobla en cantidad, al total de las películas que provienen de la Unión Europea. 


\subsection{La procedencia y duración de las películas en la cartelera:}

Las películas norteamericanas no sólo son las que predominan en las pantallas de cine de México, sino éstas también son las que se mantienen vigentes por un mayor período de tiempo en la cartelera (tabla 4). Los períodos que se mantienen en cartelera las películas norteamericanas superan por mucho a las de cualquier otra nación, incluyendo a las cintas mexicanas. De hecho, de las 26 cintas que más tiempo se mantuvieron en pantalla durante este período, 18 provienen precisamente del mencionado país.

Considerando el tiempo que se mantienen en cartelera, la mayoría de las películas, con excepción de algunas cintas provenientes de España, en realidad son pocas las naciones europeas cuyas producciones prevalecen y se mantienen por espacio de 15 o más semanas en pantalla. En el caso de Alemania y de Francia, no obstante haber exhibido una buena cantidad de cintas, tan sólo una de ellas en cada caso, se mantuvieron por un espacio de más de 10 semanas en la pantalla. En el caso de México, son dos las cintas que se encuentran entre las que mayor período de tiempo se mantuvieron en la cartelera $y$, en ninguno de los casos logran mantenerse por un espacio superior a las 15 semanas en pantalla. En este caso una de las cintas se trata de una coproducción con España y otra más de una películas totalmente mexicana.

Tabla 4

\begin{tabular}{|l|l|l|l|}
\hline & Películas & $\begin{array}{l}\text { Semanas } \\
\text { en pantalla }\end{array}$ & Origen \\
\hline 1 & Harry Potter y la cámara secreta & 24 & USA / Reino Unido \\
1 & Frida & 24 & USA \\
\hline 2 & El señor de los anillos. Las dos torres & 23 & USA / N. Zelanda \\
2 & El planeta del tesoro & 23 & USA \\
3 & Los mini campeones & 20 & USA \\
3 & Hable con ella & 20 & España \\
3 & Casarse esta en griego & 20 & USA \\
4 & El aro & 18 & USA \\
4 & Las Horas & 18 & USA \\
5 & Chicago & 17 & USA \\
5 & El hijo de la novia & 17 & Argentina y España \\
& & & \\
\hline
\end{tabular}




\begin{tabular}{|l|l|l|l|}
\hline 6 & Atrááame si puedes & 16 & USA \\
6 & Recién casados & 16 & USA \\
7 & Lucía y el sexo & 15 & España \\
7 & 8 Mile, calle de las ilusiones & 15 & USA \\
8 & Pandillas de Nueva York & 14 & USA \\
8 & Confesiones del señor Schmidt & 14 & USA \\
8 & El Rey León & 14 & USA \\
8 & Ladrón de orquídeas & 14 & USA \\
8 & El experimento & 14 & Alemania \\
8 & Lee mis labios & 14 & Francia \\
8 & Amor a segunda vista & 14 & USA \\
9 & La hija del caníbal & 13 & México/España \\
10 & Canguro Jack & 12 & USA \\
10 & Amar te duele & 12 & México \\
10 & Analízate & 12 & USA \\
& & & \\
\hline
\end{tabular}

Tabla 4 Entre las películas que más tiempo se mantienen en la cartelera predominan las cintas que son originadas en los estados Unidos de Norteamérica.

\subsection{La participación de los estudios de cine en las cartelera:}

Los estudios cinematográficos de origen norteamericano también fueron los que realizaron la mayor parte de las películas que se exhibieron en México. Principalmente en este renglón sobresale la participación de los estudios de Walt Disney y los de Buena Vista, ya que entre ambos participaron en 2 de cada 3 películas que se exhibieron durante el período de estudio (tabla 5). En el listado de los estudios que participaron de las películas que se exhibieron durante el período de estudio, no aparecen de manera significativa los europeos. Incluso, una de las obras de España que más tiempo se mantuvo en pantalla como fue la cinta "Hable con ella", es una película española, producida y dirigida por españoles, más realizada en los estudios de Sony. Empresa que tiene su sede precisamente en los Estados Unidos de América. Lo anterior pone de manifiesto que incluso las empresas norteamericanas se encuentran produciendo en otras naciones, quizá con la finalidad de evitar caer en provocaciones a las naciones que cuentan con políticas audiovisuales, como sucede en la Unión Europea. 


\section{Conclusiones:}

Tabla 5

\begin{tabular}{|l|l|l|l|}
\hline Estudios & Propia & Compartida & Total \\
\hline Walt Disney & 19 & 51 & 70 \\
Buena Vista & 16 & 51 & 67 \\
Focus Film & 25 & 3 & 28 \\
Columbia Pictures & 2 & 12 & 14 \\
Warner Brothers & 9 & 4 & 13 \\
Sony Pictures & 4 & 9 & 13 \\
Paramount & 9 & 3 & 12 \\
Miramax & 3 & 3 & 6 \\
20th Century Fox & 4 & 2 & 6 \\
Dream Works & 2 & 2 & 4 \\
\hline
\end{tabular}

Tabla 5 Los estudios que participaron con mayor cantidad de películas durante el período del análisis proceden precisamente de los Estados Unidos de Norteamérica.

Con relación al tipo de contenido, el estudio muestra que en el cine que se exhibe en las ciudades estudiadas, tiende a representar el género del drama, de la comedia y de las aventuras por encima del resto de los géneros. También se encuentra que la mayoría de los exhibidores tienden a proyectar películas que abarquen a la mayoría de las personas y que éstos además ofertarse más, son a la vez los que más tiempo permanecen en la cartelera.

En lo que hace a la procedencia de las cintas, el resultado pone de manifiesto la situación que ya se había comentado por parte de los académicos, en cuanto que la mayoría de las producciones que son presentadas proceden precisamente del mercado norteamericano. Lo anterior indica que en verdad es lamentable la situación por la que atraviesa el cine mexicano dado que las opciones que tienen los habitantes de esta misma nación para ver las producciones realizadas en su propio país en verdad son reducidas, puesto que menos del $11 \%$ de las carteleras ofrecen al público la oportunidad de presenciar el cine mexicano. Sobre todo, si la cantidad se compara con las 
producciones provenientes de otras naciones, principalmente de los Estados Unidos de Norteamérica. Esta situación coincide con los estudios realizados por Gubak y Tapio en 1982 y por Enrique Sánchez Ruiz, en 1997.

A diferencia de lo que se había mencionado, la oferta que se presenta en las carteleras del cine en las ciudades de Monterrey y México, no sólo estuvo encabezada por las que provenían de los Estados Unidos de Norteamérica. El estudio permite apreciar que también existe una notable cantidad de cintas que proceden de parte de naciones de la Unión Europea. Sin embargo, el período de tiempo que las obras provenientes de dichas naciones permanecen en la cartelera es muy corto en comparación a la duración que presentan las cintas norteamericanas. De esa forma, también son los estudios norteamericanos quienes participan de la realización de las cintas que mayor tiempo permanecen en pantalla y por ende, las que mayor oportunidad tienen de presenciar los cinéfilos mexicanos.

El cine de México, se ha convertido en un verdadero paraíso para las grandes corporaciones de cine, ya que son ellas precisamente las que vienen y no solamente nos están compartiendo de la transmisión de su cultura, sino que también éstas han sabido explotar el gran mercado nacional, lo cual es muy lamentable si se considera que nuestra nacion atraviesa precisamente por una de las situaciones de mayor dificultad de económica y de desempleo de los últimos años. En este tiempo, en que el desempleo y las bajas en la economía se han dejado sentir en México, debemos reparar de nuevo en las oportunidades para salir adelante y la industria cinematográfica ha demostrado que ésta puede ser una manera para aminorar la problemática existente. Es importante por lo pronto, que al menos se haga valer la legislación vigente en la materia y, que el gobierno refuerce los apoyos destinados a ésta ya que eso podrá contribuir al menos a aminorar la situación en que se encuentra a fin de impedir que la misma desaparezca por completo.

\section{Referencias:}

SEGOB. (2001). Ley Federal de Cinematografía y Reglamento. RTC. México.

Ugalde, Víctor. (2002) Una nueva ley. ¿Una nueva industria? Disponible en http://www.francia.org.mx/debates/noviembre/ 


\section{leydecine.htm}

Vértiz, Columba (2002). Análisis de tres generaciones de cineastas.

El cine tocó fondo. Proceso Magazine [Revista electrónica].

Diciembre 23 de 2002. México. Disponible en: http://

biblioteca.itesm.mx/cgi-bin/nav/

salta?cual=bases: $24 \&$ recargar $=62$.

Sánchez Ruíz, Enrique (2000). Industrias culturales y libre comercio. México, Canadá y la Unión Europea: hacia un Análisis comparativo de políticas de comunicación. Razón y Palabra [Revista electrónica], No. 19. Disponible en: http:// www.cem.itesm.mx/dacs/publicaciones/logos/anteriores/n19/ index.html.

Sánchez Ruíz, Enrique (2000). Industrias culturales y libre comercio. México, Canadá y la Unión Europea: hacia un Análisis comparativo de políticas de comunicación. Razón y Palabra [Revista electrónica], No. 19. Disponible en: http:/l www.cem.itesm.mx/dacs/publicaciones/logos/anteriores/n19/ index.html.

Sánchez Ruiz, Enrique. (1997) ¿Se 'norteamericaniza' el cine en México? Revista Mexicana de Comunicación [Versión electrónica]. No. 51. Disponible en: http://www.cem.itesm.mx/dacs/ buendia/rmc/rmc51/ruiz.html.

Carlos Salinas de Gortari envió a la Cámara de Diputados su proyecto de Ley de Cinematografía, el cual fue aprobado sin ningún contratiempo en el año de 1992. En la misma ley se tiende a privilegiar la liberación del mercado cinematográfico, al reducir las cuotas nacionales de $50 \%$ al $20 \%$.

Ugalde, Víctor. (2002) Una nueva ley. ¿Una nueva industria? Disponible en http://www.francia.org.mx/debates/noviembre/ leydecine.htm

Murciano Martínez, Marcial.(1992). Estructura y dinámica de la Comunicación internacional. Bosch Comunicación. Barcelona. pp. 143

Asusta el peso en taquilla (2003). Mural [Prensa electrónica]

Febrero 11. Terra Networks México S.A. de C.V. Disponible en: http://biblioteca.itesm.mx/cgi-bin/nav/ salta?cual=bases: $24 \&$ recargar $=62$.

Aguilar, Alberto. (2003). Columna financiera Nombres, nombres y ...nombres. Infosel News [En Red]. 27 de junio. Disponible en: http://biblioteca.itesm.mx/cgi-bin/nav/ 


\section{salta?cual=bases: $24 \&$ recargar $=178$}

Aguilar, Alberto. (Op. Cit.).

Casas Pérez, María de la Luz (1995). Estructura para el análisis de la reglamentación mexicana en medios audiovisuales. En Desarrollo de las industrias audiovisuales en México y Canadá. Delia Crovi Druetta (coord.). Facultad de Ciencias Políticas y Sociales. UNAM. México.

Gubak, Thomas H. y Varis, Tapio. (1982). Trasnational communication and cultural industries. UNESCO. Paris.

INEGI (2002) Estadísticas de Cultura. Cuaderno No. 6. Ags. México

Dirección General de Radio Televisión y Cinematografía. (2001). Ley Federal de Cinematografía y Reglamento. Secretaría de Gobernación. México.

Dirección electrónica de All Movie: http://www.allmovie.com. 